\title{
Diaphragmatic paralysis due to Lyme disease
}

\author{
J.L. Faul, S. Ruoss, R.L. Doyle, P.N. Kao
}

Diaphragmatic paralysis due to Lyme disease. J.L. Faul, S. Ruoss, R.L. Doyle, P.N. Kao. (C)ERS Journals Ltd 1999.

ABSTRACT: Lyme disease is a tick-borne spirochaete infection which, in a proportion of patients, can lead to neuropathy. This article describes a case of diaphragmatic paralysis due to Lyme disease.

A 39-yr-old male presented to the hospital because of an acute left facial palsy. Six weeks prior to admission he had developed a circular rash on his left flank during a camping holiday. He also complained of shortness of breath and arthralgia for 1 week. His chest radiograph demonstrated a raised right hemi-diaphragm. Diaphragmatic paralysis was confirmed by fluoroscopy (a positive sniff test). Serology revealed evidence of recent infection by Borrelia burgdorferi. On the basis of the patient's clinical presentation, a recent history of erythema migrans, and positive Lyme serology, a diagnosis of neuroborreliosis was made. He received oral doxycycline therapy (200 ${\mathrm{mg} \cdot \mathrm{day}^{-1}}^{-1}$ ) for three weeks. Facial and diaphragmatic palsies resolved within eight weeks.

On the basis of this case, a diagnosis of Lyme disease should be considered in patients from endemic regions with otherwise unexplained phrenic nerve palsy. Eur Respir J 1999; 13: 700-702.

Lyme disease is a multisystem tick-borne infectious disease, caused by a spirochaete, Borrelia burgdorferi $[1$, 2]. Several species of ticks, with a very wide geographical distribution, are thought to transmit the disease to humans. Vectors of Lyme disease include Ixodes ricinus in Europe, I. pacificus in northern California, and I. scapularis in the eastern USA [1-4]. The most characteristic clinical marker of the disease, erythema migrans, has an incidence of approximately $40-70$ per 100,000 in some parts of Europe [2-5]. Although skin rashes, cardiac conduction defects and neuropathies are commonly reported features, serious respiratory complications of Lyme disease are uncommon [4]. To the authors' knowledge, this is the first description of unilateral diaphragmatic paralysis as a complication of Lyme disease.

\section{Case report}

A 39-yr-old male presented at the hospital with left facial weakness. He had developed a skin rash on his left flank six weeks previously, while on a camping holiday in a wooded area in County Galway, Ireland. This rash started as a coin sized area of redness, but over a period of 4 days progressed to a large ring of erythema (10 $\mathrm{cm}$ in diameter). The rash gradually resolved during the next 10 days. One week prior to admission he complained of right shoulder and bilateral knee pains and mild shortness of breath. He complained of dyspnoea that was not worse on exertion and he denied orthopnoea. On the morning of admission he developed weakness of the left-side of his face. There was no headache, hyperacusis, neck stiffness or visual symptoms. There was no cough or wheeze. The patient was an otherwise healthy classical guitarist. He took no medication and did not abuse tobacco or alcohol. He was allergic to penicillin.
On physical examination the patient was not febrile. The pupils, eye movements, visual acuity and fundoscopy were normal. The neck was supple with no palpable glands or nodes. The patient was unable to blink, frown or grimace on the left-side of the face. The eardrums appeared normal. The right lung base was dull to percussion, with absent breath sounds. The right shoulder and both knees appeared normal and demonstrated a full range of movement. There was no evidence of joint effusion.

The chest radiograph (fig. 1) demonstrated an elevated right hemi-diaphragm. Fluoroscopic screening of diaphragmatic movement during "sniff" manoeuvres revealed paradoxical movement of the right hemi-diaphragm, consistent with a right phrenic nerve paralysis. An electrocardiograph was normal. The patient refused to undergo computerized spirometry and phrenic nerve stimulation testing. Arterial blood gases (patient supine) revealed $\mathrm{pH}$ $=7.40$, arterial oxygen tension $\left(P_{\mathrm{a}}, \mathrm{O}_{2}\right)=14.2 \mathrm{kPa}$, arterial $\mathrm{CO}_{2}$ tension $\left(P \mathrm{a}, \mathrm{CO}_{2}\right)=3.6 \mathrm{kPa}, \mathrm{HCO}_{3}{ }^{-}=26 \mathrm{mmol} \cdot \mathrm{L}^{-1}$, oxygen saturation $=99 \%$. Antinuclear antibodies, rheumatoid factor, Venereal Disease Research Laboratory (VDRL) test for syphilis, antistreptolysin-O test (ASOT) and antiacetylcholine receptor (AChR) antibodies were negative. Cerebrospinal fluid was not examined. Immunoglobulin (Ig)M and IgG antibodies to B. burgdorferi were detectable in serum (by both indirect immunofluorescence and enzyme-linked immunosorbent assay (ELISA) with purified antigen and Western blot analysis).

In the setting of erythema migrans six weeks earlier, an acute onset of arthralgia and nerve palsies, and serology demonstrating recent infection with $B$. burgdorferi, a diagnosis of Lyme disease was made. The diagnosis of diaphragmatic paralysis was made on the basis of clinical signs and chest radiography. The patient received oral 

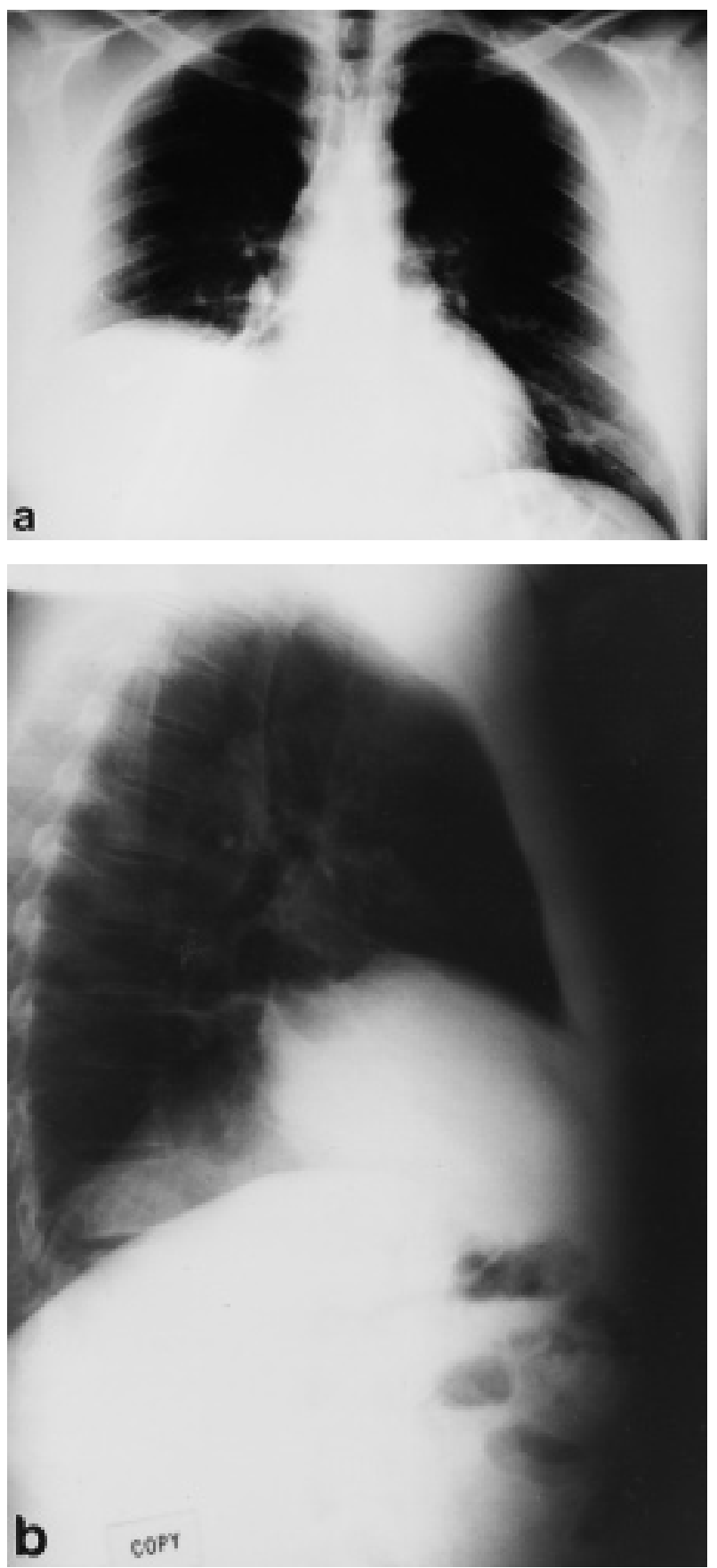

Fig. 1. - Chest radiographs in a) posteroanterior and b) right lateral positions, demonstrating a raised right hemi-diaphragm.

doxycycline (200 mg. day ${ }^{-1}$ for 3 weeks) as therapy. Within 5 days the facial weakness had resolved. After 8 weeks the chest radiograph was normal and he demonstrated a full return of diaphragmatic movement with inspiration. He remains symptom-free $1 \mathrm{yr}$ later.

\section{Discussion}

In this case, diaphragmatic paralysis due to Lyme disease was diagnosed on the basis of a history of shortness of breath, chest radiography demonstrating a raised and paralysed right hemi-diaphragm, clinical features and serology indicating recent infection by $B$. burgdorferi, and a rapid and complete response to appropriate antibiotic therapy.

Because the ticks that transmit Lyme disease are commonly found in wooded areas or parkland, patients often give a history of outdoor activities $[1,2]$. As in this case, there is no history of tick bite in the majority of cases [6]. In Europe approximately $80 \%$ of patients with Lyme disease develop erythema migrans [2]. Erythema migrans usually spontaneously resolves within 2 weeks. However, if left untreated, Lyme disease may lead to progressive neurological and arthritic complications in at least a small proportion of cases. The current patient presented because of a facial palsy. Paralysis of the facial nerve is particularly common in Lyme disease and has been reported to occur in approximately $3 \%$ of patients [7]. Various other neurological sequelae of Lyme disease have been described, including aseptic meningitis, transverse myelitis and mononeuritis multiplex $[1,8,9]$. Lyme disease may cause paralysis of multiple nerve roots and multisegmental intercostal nerves [10]. Cases of respiratory failure requiring tracheostomy and ventilatory support (owing to an impaired central respiratory drive) have also been reported [11]. However, this patient is, to the authors' knowledge, the first case of diaphragmatic paralysis due to Lyme disease.

Unilateral diaphragmatic paralysis is a serious respiratory disorder and many significant neurological diseases, such as spinal cord injury, motor neuron disease, and carcinomatous infiltration of the phrenic nerve, are included in its differential diagnosis. This article describes the development of diaphragmatic paralysis in a patient with Lyme disease. He did not demonstrate evidence of respiratory failure (even when supine), he did not complain of orthopnoea, and he denied any reduction in exercise tolerance. Perhaps it is not surprising that there was no evidence of respiratory failure in this case since, in the absence of underlying lung disease, unilateral diaphragmatic paralysis is a rare cause of respiratory failure and its symptoms can be mild $[12,13]$.

Oral tetracycline antibiotics are the treatment of choice for all stages of Lyme disease. Similar clinical cure rates are seen with oral doxycycline for 3 weeks compared with intravenous ceftriaxone for 2 weeks [14]. Because of better penetration into cerebrospinal fluid, intravenous ceftriaxone is recommended for patients with meningitis or encephalopathy, but for most patients with Lyme disease intravenous therapy appears no more effective than oral therapy and may be more likely to result in complications [14]. Ceftriaxone appears to be an effective alternative to tetracycline therapy and is especially useful in young children and pregnant females. The patient in this case report was severely allergic to penicillin and refused therapy with cephalosporin antibiotics, and he was accordingly prescribed oral doxycycline.

This case study describes diaphragmatic paralysis due to Lyme disease. Oral doxycycline therapy proved effective in reversing the clinical and radiological features. Lyme disease should be considered a differential diagnosis in patients from endemic areas with unexplained phrenic nerve palsy. 


\section{References}

1. Reik L. Lyme disease. In: Scheld WM, Whitley RJ, Durack DT, eds. Infections of the Central Nervous System, 2nd Edn. Philadelphia, Lippincott-Raven, 1997; pp. 685-718.

2. Berglund J, Eitrem R, Ornstein K, et al. An epidemiologic study of Lyme disease in southern Sweden. $N$ Engl J Med 1995; 333: 1319-1327.

3. Hansen K, Lebech AM. The clinical and epidemiological profile of Lyme neuroborreliosis in Denmark 1985-1990. A prospective study of 187 patients with Borrelia burgdorferi specific intrathecal antibody production. Brain 1992; 115: 399-423.

4. Nadelman RB, Nowakowski J, Forseter G, et al. The clinical spectrum of early Lyme borreliosis in patients with culture-confirmed erythema migrans. $\mathrm{Am} \mathrm{J} \mathrm{Med}$ 1996; 100: 502-508.

5. de Mik EL, van Pelt W, Docters-van Leeuwen BD, van der Veen A, Schellekens JF, Borgdorff MW. The geographical distribution of tick bites and erythema migrans in general practice in the Netherlands. Int $J$ Epidemiol 1997; 26: 451-457.

6. Kuiper H, Cairo I, Van Dam A, et al. Solitary erythema migrans: a clinical, laboratory and epidemiological study of 77 Dutch patients. Br J Dermatol 1994; 130: 466-472.
7. Gerber MA, Shapiro ED, Burke GS, Parcells VJ, Bell GL. Lyme disease in children in southeastern Connecticut. Pediatric Lyme Disease Study Group. $N$ Engl J Med 1996; 335: 1270-1274.

8. Krishnamurthy KB, Liu GT, Logigian EL. Acute Lyme neuropathy presenting with polyradicular pain, abdominal protrusion and cranial neuropathy. Muscle Nerve 1993; 16: $1261-126$

9. Halperin JJ. Neuroborreliosis: central nervous system involvement. Semin Neurol 1997; 17: 19-24.

10. Roche C, Durieux A, Clavelou P. Pseudo-eventration abdominale revelant une maladie de Lyme. Annales de Medecine Interne (France) 1997; 148: 188-189.

11. Silva MT, Sophar M, Howard RS, Spencer GT. Neuroborreliosis as a cause of respiratory failure. $J$ Neurol 1995; 242: 604-607.

12. Newsom-Davis J. The diaphragm and neuromuscular disease. Am Rev Respir Dis 1979; 119: 115-117.

13. Mulvey DA, Aquilina RJ, Elliott MW, Moxham J, Green M. Diaphragmatic dysfunction in neuralgic amyotrophy: an electrophysiologic evaluation of 16 patients presenting with dyspnea. Am Rev Respir Dis 1993; 147: 66-71.

14. Eckman MH, Steere AC, Kalish RA, Pauker SG. Costeffectiveness of oral as compared with intravenous antibiotic therapy for patients with early Lyme disease or Lyme arthritis. N Engl J Med 1997; 337: 357-363. 